\title{
An Automatic Method for Enhancing the Display of Different Tissue Densities in Digital Chest Radiographs
}

\author{
Michael F. McNitt-Gray, Ricky K. Taira, Sandy L. Johnson, and Mahmood Razavi
}

\begin{abstract}
Digital chest radiographs are often too bright and/or lack contrast when viewed on a video display. This often occurs in radiographs taken of patients with dense lungs, or when incorrect $x$-ray exposure techniques or inappropriate image preprocessing operations are performed (eg, by the computed radiography system or laser scanner). This article describes a method to automatically provide brightness and contrast adjustments to selectively enhance either soft or dense tissues. This method reduces viewer interaction and improves displayed image quality. The algorithm analyzes the gray-level histogram of a chest radiograph and determines the breakpoints that separate the region outside the patient (background), the radiographically soft tissues, and the radiographically dense tissues. From these breakpoints, a series of piecewise linear look-up tables (LUTs) is generated to selectively enhance either the soft tissues or the dense tissues. This is performed by: (1) varying the contrast in the patient background to achieve the desired overall brightness, (2) selectively increasing the contrast of the tissue region of interest, and (3) reducing or maintaining the contrast of the remaining region. The resulting LUTs are applied to the original image via video display.
\end{abstract}

Copyright 1993 by W.B. Saunders Company

KEY WORDS: image processing, image display, look-up tables, chest radiography, picture archiving and communication system.

$\mathbf{I}^{\wedge}$ A PICTURE archiving and communication system (PACS $)^{1-8}$ chest radiographs are acquired, archived to long-term storage, and routed to a high-resolution display workstation, all in digital form. At the display workstation, the radiologist reviews the image on a video display monitor. Chest images displayed on the monitors are often too bright or lack sufficient contrast. This can be due to many factors: the exposure technique may not sufficiently penetrate the different tissues (especially pediatric patients from the neonatal intensive care unit with underdeveloped lungs); the brightness and contrast improvements performed by the image acquisition device may not be optimal (ie, they may be designed for some "standard" chest image and may not truly apply to a specific image); or the image acquisition devices may drift out of calibration and produce very bright or poor contrast images.

Presently, radiologists have a limited number of options when faced with a video image that is too bright and/or lacks contrast. These options include direct image interaction through analog brightness and contrast knobs or interactive digital level and window operations. When the viewer adjusts the brightness and contrast knobs, the adjustments must be made for each image. This creates nonstandard viewing conditions and requires frequent viewer interaction. Interactive digital level and window adjustments may be used, but this requires even greater viewer interaction.

Several automatic preprocessing methods have been proposed to improve the displayed contrast of digital images. ${ }^{9}$ These methods include histogram equalization, unsharp masking, and variations of the two. Histogram equalization ${ }^{10}$ can be applied to digital chest images automatically to enhance areas of low contrast, but it may enhance the background at the expense of the areas of interest. Adaptive histogram equalization $(\mathrm{AHE})^{11}$ enhances areas of interest by using histogram equalization that is locally adaptive. Extensions to the basic AHE method have included limits on the enhancement (contrast-limited AHE) ) $^{12}$ and the suppression of artifacts by the calculation and subtraction of a background (artifact-suppressed AHE). ${ }^{13}$ Although these methods are successful in improving the contrast in the images, they are computationally intensive and yield only one image. This gives the viewer little flexibility in selecting the desired brightness and contrast settings.

Several other methods have been proposed to

From the Department of Radiological Sciences, University of California, Los Angeles, CA.

Supported in part by Public Health Senice Grant nos. T32 GM 08375 and POI CA 51198 awarded by the National Institute of General Medical Sciences and the National Cancer Institute, respectively.

Address reprint requests to Michael F. McNitt-Gray, MS Department of Radiological Sciences, University of California, Los Angeles, AR-277 CHS, 10833 Le Conte Ave, Los Angeles, CA 90024-1721.

Copyright 1993 by W.B. Saunders Company

0897-1889/93/0602-0001\$3.00/0 
account for the different processing requirements of the lung and mediastinum areas. McAdams et al ${ }^{14}$ have described a method that analyzes the image histogram to design gray scale modification look-up tables (LUTs) as well as an anatomy-sensitive high frequency emphasis parameter for adaptive unsharp masking. This histogram-directed method gives impressive results; however, it derives these histograms interactively and is very operator intensive. Sherrier and Johnson ${ }^{15}$ have described a method that determines whether each pixel under consideration is in the heart, mediastinum, or subdiaphragm region. For each region, a separate form of AHE is performed. Sezan et $a^{16}$ have described a method in which heuristic rules have been applied to the image histogram to identify the lung/mediastinum gray level threshold. Based on the threshold obtained, a high frequency emphasis parameter is determined for use in adaptive unsharp masking. This article describes a method that uses heuristic rules (different from those in Sezan et $\mathrm{al}^{16}$ ) to determine several regions within the gray level histogram. However, neither AHE or unsharp masking are used; instead, several gray level modification LUTs are created to give the viewer more flexibility in viewing digital chest images.

This method automatically creates several piecewise linear LUTs that allow the viewer to selectively enhance different tissue densities in digital chest images. This is accomplished by analyzing the histogram of the image to determine several key breakpoints. Based on these breakpoints, three regions of interest can be identified: (1) the background region, (2) the radiographically soft tissue region and (3) the radiographically dense tissue region. Using the breakpoints, we can apply different gains to increase the contrast (gain $>1$ ) or reduce the contrast (gain $<1$ ) of each region individually. In this way, the brightness and contrast of each region can be adjusted. The result is a series of piecewise linear LUTs that are created to enhance each region of interest selectively. Nine different LUTs are generated: one linear LUT with no enhancement, two LUTs that enhance the radiographically soft tissues (with different amounts of gain) and six LUTs that enhance the radiographically dense tissues (each with different amounts of gain). Thus, with a minimum amount of viewer interaction, either the more soft or more dense tissues can be enhanced by applying the precalculated LUTs. This method has been implemented in a clinical PACS at the University of California at Los Angeles.

\section{METHODS AND MATERIALS}

\section{Obtaining the Image Histogram}

Digital chest radiographs in our PACS are acquired and sent to a central file server and archive (known as a cluster controller), where the image is subsampled to generate a gray level histogram. Figure 1 shows a typical digital pediatric chest image and Fig 2 shows the corresponding histogram of this image. Figure 2 shows the "noisy" nature of the histogram. This makes the determination of significant breakpoints (ie, the boundaries between pixel populations) difficult. The histogram is smoothed using a local average to aid in the location of these breakpoints.

\section{Identification of Breakpoints}

Examination of the smoothed histograms shows a distinct pattern that is observed in most chest images. This leads to the hypothesis that the histogram of a typical chest has three distinct regions that include (Fig 3) (1) A background region, (2) A radiographically soft tissue region, and (3) A radiographically dense tissue region. The background region is characterized by a peak in the lowest gray levels. This region is caused by areas that are outside the patient but within the radiation field. These are areas with little or no attenuation of the $x$-ray beam and have the darkest (lowest) pixel values. The soft tissue region begins with the gray
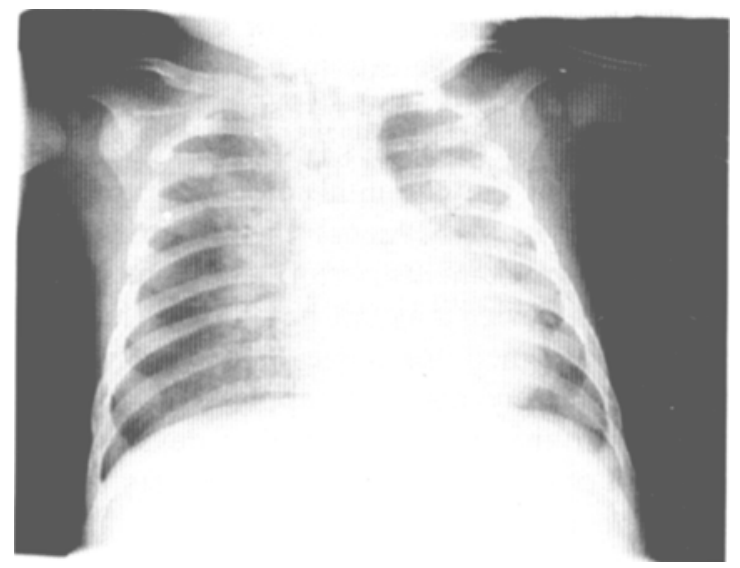

Fig 1. Typical digital image of a pediatric chest. 


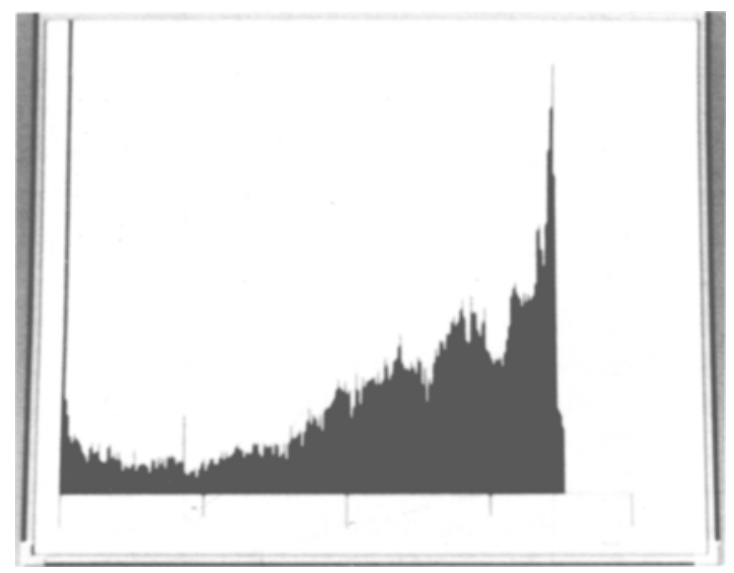

Fig 2. Actual gray level histogram of image in Fig 1.

levels that are brighter than the background region and have a low number of occurrences without any peak in the histogram. This region contains the softest tissues in the chest image and may include skin, muscle, and sometimes the less dense portions of lung tissues (those that are well-penetrated by the $x$-ray beam). Pixels belonging to the lung region often have gray values brighter than the softest tissues, continuing up to approximately the image average gray value. The lung region is considered to be part of the soft tissuc region. The dense tissue region is characterized by gray levels that are brighter than the soft tissue region and are contained in a large, broad peak. This region contains the remaining tissues including those of the heart and mediastinum, subdiaphragm,

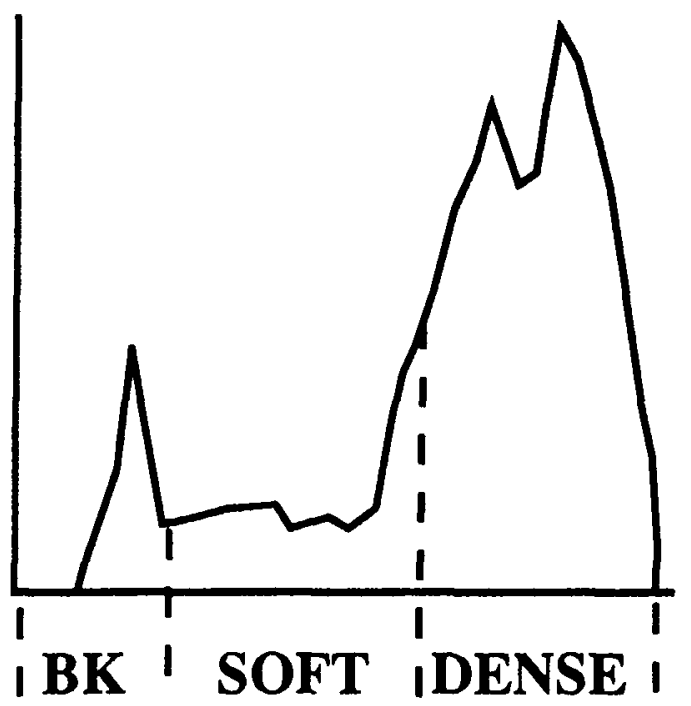

Fig 3. Definition of the three regions in a typical digital chest image histogram. and the densest lung tissues (those that are not well penetrated).

Several heuristic rules were created to identify these three regions from the smoothed image histogram. These rules describe how to locate the breakpoints that identify the regions. The first breakpoint (point $A$ in Fig 4 ) is the first gray level (greater than gray level 0 ) that has a non-zero frequency of occurrence. This is the beginning of the background region. The next breakpoint (point $B$ in Fig 4 ) is the gray level that has the first local maximum following point $A$. The third breakpoint (point $C$ in Fig 4 ) is the first local minimum following the maximum. This defines the end of the background region and the beginning of the soft tissue region. The next breakpoint (point $D$ in Fig 4) is the average gray value of the image. This point describes the end of the soft tissue region and the beginning of the dense tissue region. Pietka et al ${ }^{17}$ used the average gray level as a threshold for the lung region (ie, most of the lung region has gray values less than or equal to the average gray value). Finally, breakpoint $E$ is the last gray level with a non-zero frequency of occurrence. This point describes the end of the dense tissue region.

The heuristic rules described above are used to find all of the breakpoints that identify the three regions. The background region consists of gray levels from 0 (zero) to breakpoint $C$. The radiographically soft tissue region consists of gray levels from breakpoints $C$ to $D$. The radio-

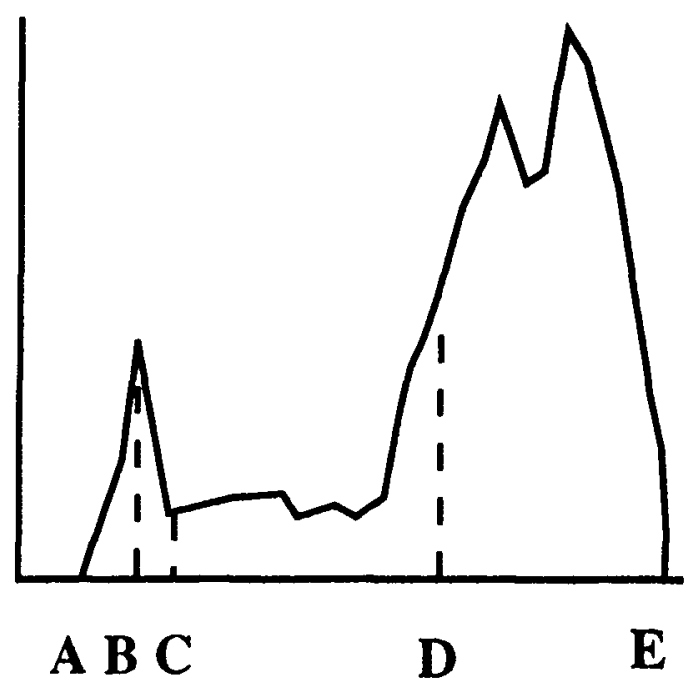

Fig 4. Breakpoints in a typical digital chest image histogram. 
graphically dense tissue region consists of gray levels from breakpoints $D$ to $E$. Using this information, piecewise linear LUTs are created.

\section{Create Piecewise Linear LUTS}

Once the three regions are identified, LUTs that enhance each region separately are created. This is done by increasing the contrast for the region of interest and adjusting the contrast for the remaining regions. For an LUT, this consists of assigning gains of greater than one to increase contrast or less than one to decrease contrast. By varying the gain of each region separately, a piecewise linear LUT is created as shown in Fig 5. This figure shows the creation of an LUT that enhances the dense tissue region. When such an LUT is applied to the original image, a remapping of gray levels occurs as shown in Fig 6.

This must be done within the limits of the gray levels available for display and within the useful range of gray levels. The display monitors used in our PACS can display 256 gray levels ( 8 bits). The LUT creation step must take these factors into account as gray levels are reallocated.

In this analysis, there are three regions and each region is assigned a gain to create the LUT. Therefore, we use the following definitions:

$A_{b}$, gain applied to the background region

$A_{s}$, gain applied to the radiographically soft tissue region

$A_{4}$, gain applied to the radiographically dense tissue region

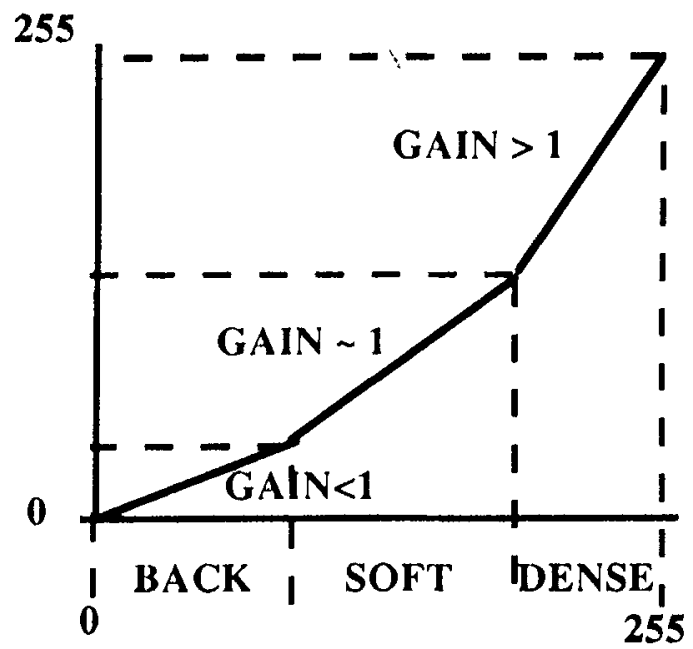

Fig 5. Example of an LUT to enhance the dense tissue region.

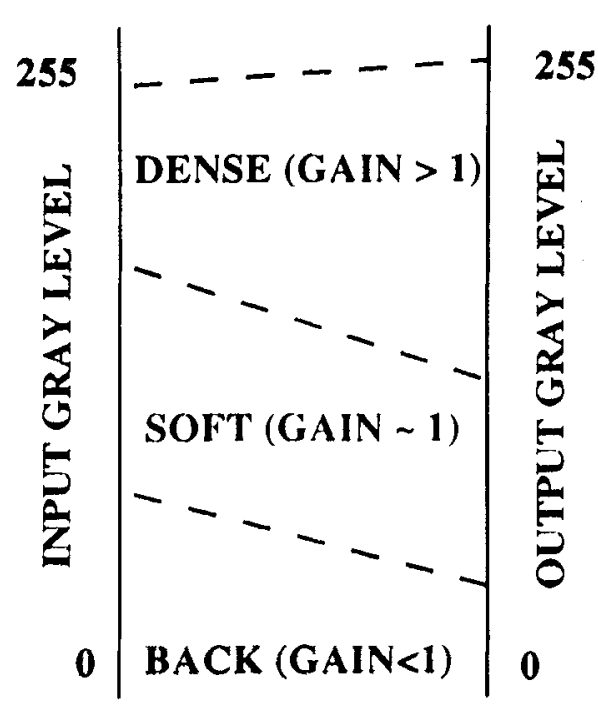

Fig 6. Mapping of gray levels when the LUT of Fig 5 is applied.

$N_{b}$, number of gray levels occupied by the background region

$\mathrm{N}_{\mathrm{s}}$, number of gray levels occupied by the soft region

$N_{\mathrm{d}}$, number of gray levels occupied by the dense region

$Z_{\max }$, the highest output gray level allowed (typically 255 for 8-bit image)

$Z_{\min }$, the lowest output gray level allowed (typically 0 )

LUT[i], the LUT array

i, gray level

A, B, C, D, E, breakpoints as described above.

The LUT is created by applying a different gain to each region in the following manner:

$$
\begin{array}{rlrl}
\operatorname{LUT}[i] & =\left(A_{b} * i\right)+Z_{\min } & & 0 \leq i \leq C \\
\operatorname{LUT}[i] & =\left(A_{s} *(i-C)\right)+\left(A_{b} * C\right)+Z_{\min } & C<i \leq D \\
\operatorname{LUT}[i] & =\left(A_{d} *(i-D)\right)+\left(A_{s} *(D-C)\right) & & D<i \leq E \\
& \quad+\left(A_{b} * C\right)+Z_{\min } & & E<i \\
\operatorname{LUT}[i] & =Z_{\max } & &
\end{array}
$$

In the next section, we discuss how each of the gains are determined.

\section{Enhancing the Radiographically Dense Tissue Region}

To enhance the dense region, the dense tissue region should have its contrast increased and the overall brightness of the image should be decreased. To accomplish this, a three-step process is performed. First, a gain of greater than one is specified for the dense region to increase contrast. Next, the gain of the soft tissue region is maintained at one, if possible, because this region usually has good contrast (and no enhancement may be necessary). Finally, the background region has a gain of less 
than one to reduce its contrast. This reduces the overall brightness of the image. This three-step process is repeated for all of the desired dense gain values.

For chest images, the values of the dense region gain are assigned values from 1.2 to 2.2. These values were empirically determined to give a good range of contrast enhancements. Gains higher than 2.2 often provided too much contrast and were not found to be useful. The number of output gray levels that the dense region will occupy is found by multiplying the specified gain $\left(A_{b}\right)$ by the input dense region range of gray levels $(E-D)$ :

$$
\begin{aligned}
& N_{d}=A_{d} *(E-D) \\
& \text { If } \left.\left[N_{d}>\left(Z_{\max }-Z_{\min }\right)\right] \text { (ie, if allocated }>\text { available }\right) \text {; } \\
& \text { then reduce } A_{d} \text { until } N_{d}<\left(Z_{\max }-Z_{\min }\right) \text {. }
\end{aligned}
$$

This also determines the number of gray levels left to be allocated to the soft and background regions. If the dense gain is too high, there may not be a sufficient number of gray levels to maintain soft tissue contrast and soft tissue contrast may be reduced:

$$
N_{\mathrm{s}}=A_{\mathrm{s}} *(D-C) \text {, where } A_{\mathrm{s}}=1 \text { : }
$$

However, if $\left[\left(N_{\downarrow}+N_{s}\right)>\left(Z_{\max }-Z_{\text {min }}\right)\right]$ (if allocated $>$ available); then (reduce $A_{s}$ until $\left(N_{d}+N_{s}\right) \leq$ $\left(Z_{\max }-Z_{\min }\right)$, and set $\left.A_{b}=0\right)$

If there are still gray levels to be allocated (ie, if $\left(N_{d}+N_{s}\right)<\left(Z_{\max }-Z_{\text {min }}\right)$ ), the gain for the background region is calculated as the following equation:

$$
A_{b}=\left[\left(Z_{\max }-Z_{\min }\right)-\left(N_{d}+N_{s}\right)\right] / C
$$

In this way, the gain for each region can be determined after specifying the dense tissue region gain. As the dense tissue gain is increased, the contrast of this region is increased. As the background region contrast is reduced, this darkens the entire image. This is shown in the example LUT in Fig 5 and the resulting gray-level mapping shown in Fig 6.

\section{Enhancing the Radiographically Soft Tissue Region}

To enhance the soft tissue region, a similar set of steps are undertaken. The gain of the soft tissue is specified to be greater than one (soft gain values of 1.2 and 1.4 are used in this report because gains higher than this have not been shown to be useful from our preliminary clinical assessments). The range of gray levels that the soft tissue region will occupy can be calculated as above. This will result in the reduction of contrast for the dense tissue region. In the case of very soft tissues (those with very low gray levels) this increase in contrast may still not make them appear with sufficient brightness. For this reason, the background regionusually a region that occupies a limited number of gray levels-also has its contrast increased to brighten the image. Thus, the gain specified for the soft tissue region will also be applied to the background region in this case. The following equations are used to find the appropriate gain for the dense region:

$$
\begin{aligned}
& N_{\mathrm{s}}=A_{\mathrm{s}} *(D-C) \\
& N_{\mathrm{b}}=A_{b} *(C) \\
& N_{\mathrm{g}}=\left[\left(Z_{\text {max }}-Z_{\text {min }}\right)-\left(N_{\mathrm{b}}+N_{\mathrm{s}}\right)\right] /(E-D)
\end{aligned}
$$

An example of a soft tissue enhancement LUT and its mapping are shown in Figs 7 and 8.

\section{Implementation in a PACS}

The PACS consists of several major components. ${ }^{1-8}$ Among these are the image acquisition devices. In our PACS, digital radiographs are acquired from three computed radiography (CR) systems (one Philips Computed Radiography system PCR-901 and two PCR-7000, Philips Medical Systems, Shelton, CT) and a laser scanner (Konica Laser Scanner Model KLSR-S, Konica, Tokyo, Japan). These images are refor-

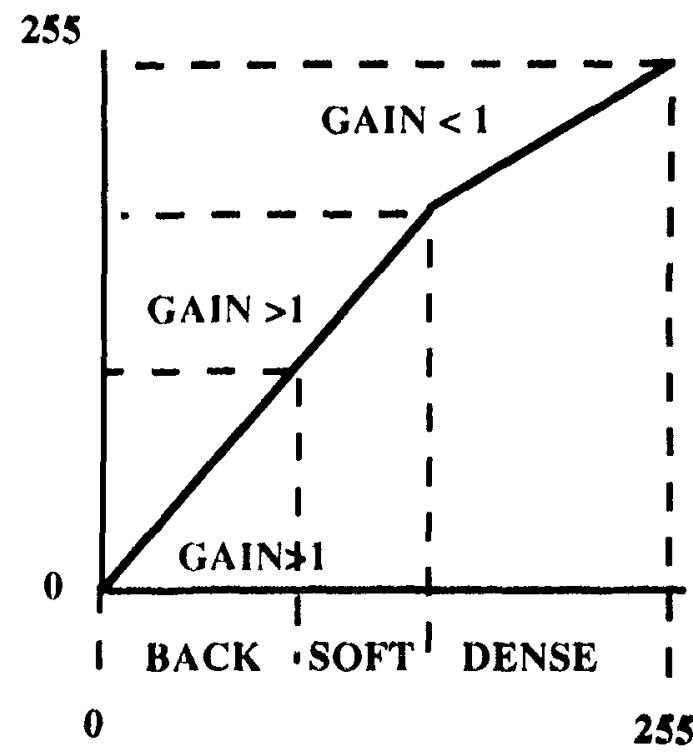

Fig 7. Example of an LUT to enhance the soft tissue region. 


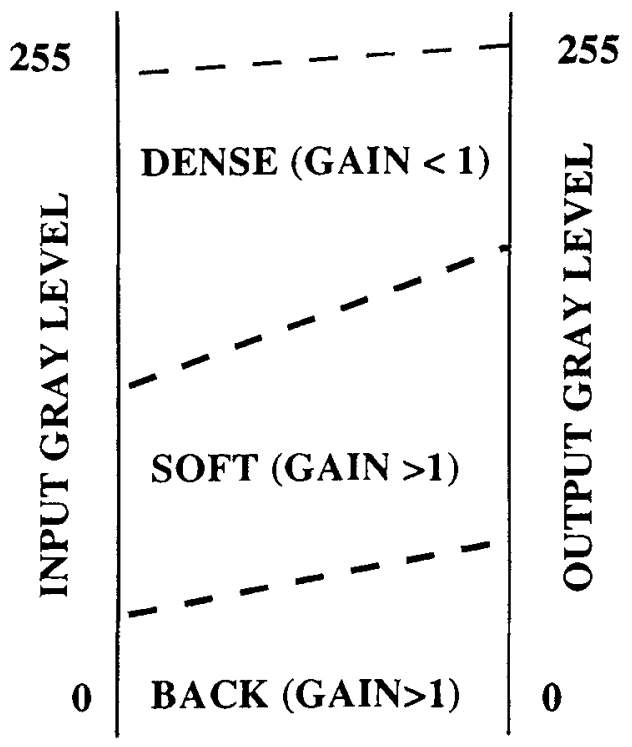

Fig 8. Mapping of gray levels when the LUT of Fig 7 is applied.

matted on an acquisition computer (Sun 4/370, Sun Microsystems Inc, Mountain View, CA) and sent to a cluster controller computer.

At the cluster controller computer each image is automatically sampled to create the histogram and the breakpoint analysis (described above) is performed on each image individually. The resultant breakpoints are stored in the header of the image file for later use. The image and header are long-term archived onto an optical disk library, and a copy is also routed to the appropriate display workstation where it is stored on parallel transfer disks.

At the display workstation, as the image is read from the parallel transfer disks, the breakpoints are read from the header information and nine piecewise linear LUTs are calculated automatically. The LUTs created are as follows: six for increased dense tissue contrast, two for increased soft tissue contrast, and one linear LUT (no enhancement, to re-create the original). By the time the image is available for display, all of the LUTs have been created and are ready for use.

Because the display routines are written in the $\mathrm{X}$-window environment, ${ }^{8}$ the viewer can select which tissue region to enhance by clicking a cursor on the appropriate icon. One icon applies the LUT of the next higher dense tissue gain (or lower soft tissue gain). Another icon applies the LUT of the next lower dense tissue gain (or higher soft tissue gain). In this way, the viewer can step through the sequence of LUTs rather than being limited to a single LUT.

\section{RESULTS}

For the image in Fig 1, all five breakpoints were calculated: $\mathrm{A}=1, \mathrm{~B}=8, \mathrm{C}=25, \mathrm{D}=132$, and $\mathrm{E}=230$ (all units are gray level values). The algorithm described above assumes that the background region is from breakpoint $\mathrm{A}$ to $\mathrm{C}$ (gray level 0 to 25), the soft tissue region is from $\mathrm{C}$ to $\mathrm{D}$ (gray levels 26 to 132 ) and that the dense tissue region is from D to $\mathrm{E}$ (gray levels 133 to 230 ). To show that the breakpoints do correspond to the assumed regions of radiographic density, the original image in Fig 1 has been thresholded at each of the above mentioned values. Figure 9A shows the original image which has been thresholded between gray values 0 to 25 . This gray level range contains only pixels belonging to the background region outside the patient. Figure 9B shows the original image thresholded between gray levels 26 to 132. This gray level range contains the pixels belonging to muscle, fat, skin and lung regions whereas the densest part of the lungs has not been included. Figure $9 \mathrm{C}$ shows the original image thresholded between gray levels 133 to 230. This gray level range contains the densest part of the lungs as well as the mediastinum, subdiaphragm, and dense bone.

Six dense tissue gains $(1.2,1.4,1.6,1.8,2.0$, and 2.2), two soft tissue gains (1.2 and 1.4) and one linear LUT (to re-create the original) have been used. For the purposes of reporting results, only one image is considered and a representative sample of the different LUTs are displayed here. Figure 10 shows the original and processed images for one clinical pediatric chest image.

Comparing the original image in Fig $10 \mathrm{~A}$ to the increased soft tissue gain image of Fig 10B shows the improvement is limited to two areas. The first improvement is in the soft tissue areas, such as skin, muscle, and fat. The other improvement may occur where the lung is over penetrated, making it too dark to be very visible in the original image. The brightening of the image and the increased gain of the soft tissue 


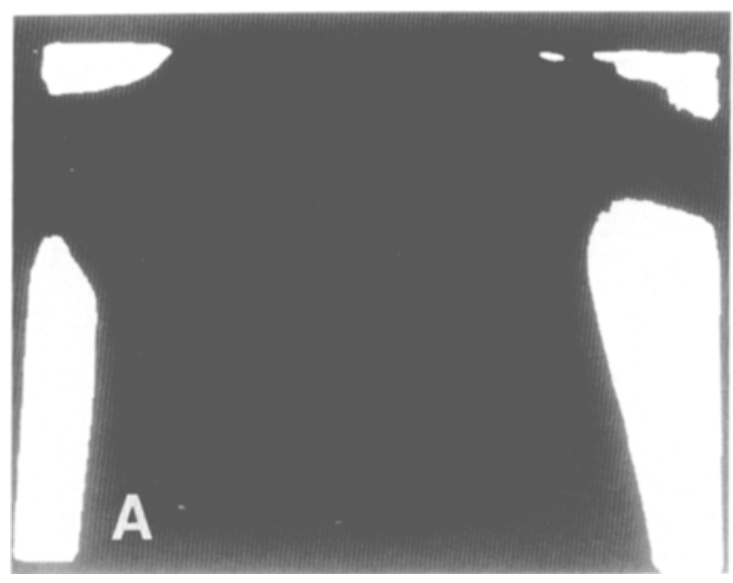

Fig 9. (A) Thresholded image of Fig 1 showing background region in white. The image has been thresholded between gray levels 1 and 25. (8) Thresholded image of Fig 1 showing the radiographically soft tissue region in white. The image has been thresholded between gray levels 26 and 132. (C) Thresholded image of fig 1 showing the radiographically dense tissue region in white. The image has been thresholded between gray levels 133 and 230.

region allows these structures to become more visible. These improvements are obtained at the expense of reduced contrast and increased brightness of the denser tissues.

Comparing Figs $10 \mathrm{C}$ and $10 \mathrm{D}$ with the original image in Fig $10 \mathrm{~A}$ shows the contrast enhancement of the dense tissues and the darkening of the image as the gain is increased. This results in improved visualization of the denser structures of the chest. However, this improvement is accomplished at the expense of decreased contrast and decreased brightness of the softer tissues.

As a result of the increased gain in the piecewise linear LUTs, the contrast between selected gray levels is increased. If the contrast enhancement is large enough, this may lead to an enhancement of the image noise as well. To demonstrate this effect, we have used a chest phantom under extremely low exposure conditions to create a very noisy image. The original (no LUT applied) is shown in Fig 11A. This
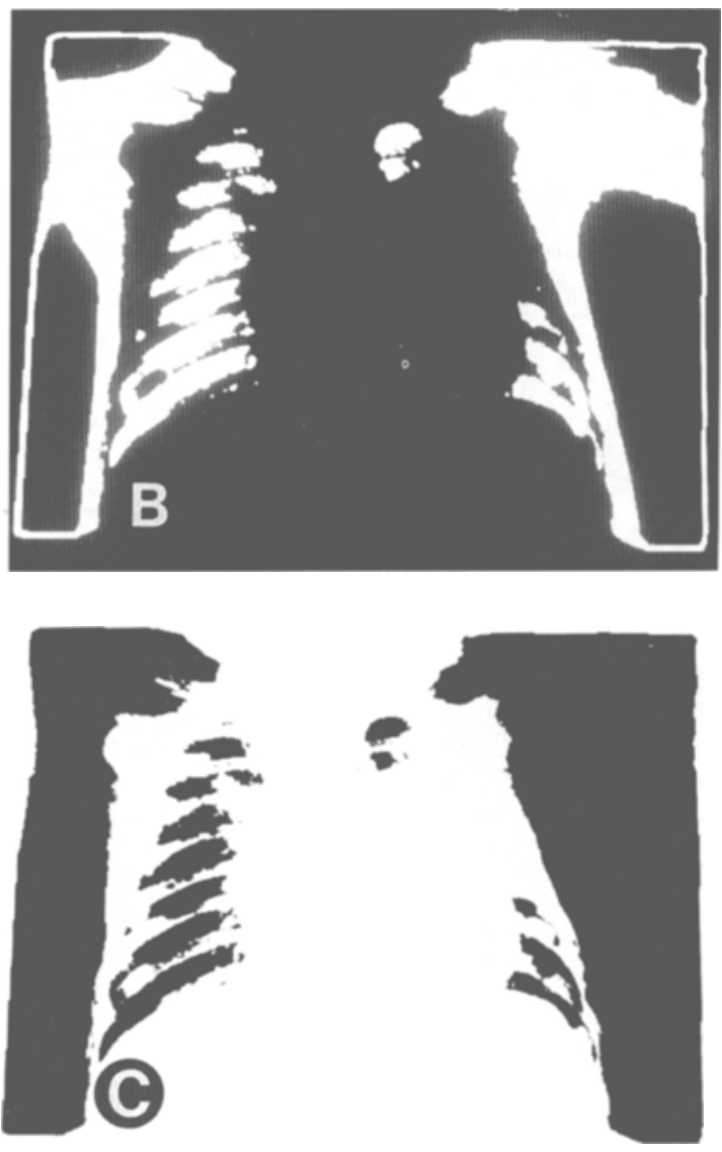

image was analyzed using the methods described above and piecewise linear LUTs were created. The LUT with the largest dense gain ( $\mathrm{dg}=2.2$ ) was applied and the result is shown in Fig $11 \mathrm{~B}$. Comparing these two images, we can see an increase in the noise in Fig 11B. This is because of the contrast enhancement introduced by the LUT. The contrast of the tissues has been increased as well as the contrast between the noise. Though this may be a problem at high noise levels and high LUT gains, it does not appear to be significant for clinical images at the levels of enhancement discussed in this report (this effect is not apparent in Figs $10 \mathrm{C}$ or $10 \mathrm{D})$.

\section{DISCUSSION}

Applying multiple piecewise linear LUTs has allowed superior visualization of the dense tissues and associated structures, especially in images that are too bright and/or lack contrast. The structures of the mediastinum, the retrocar- 

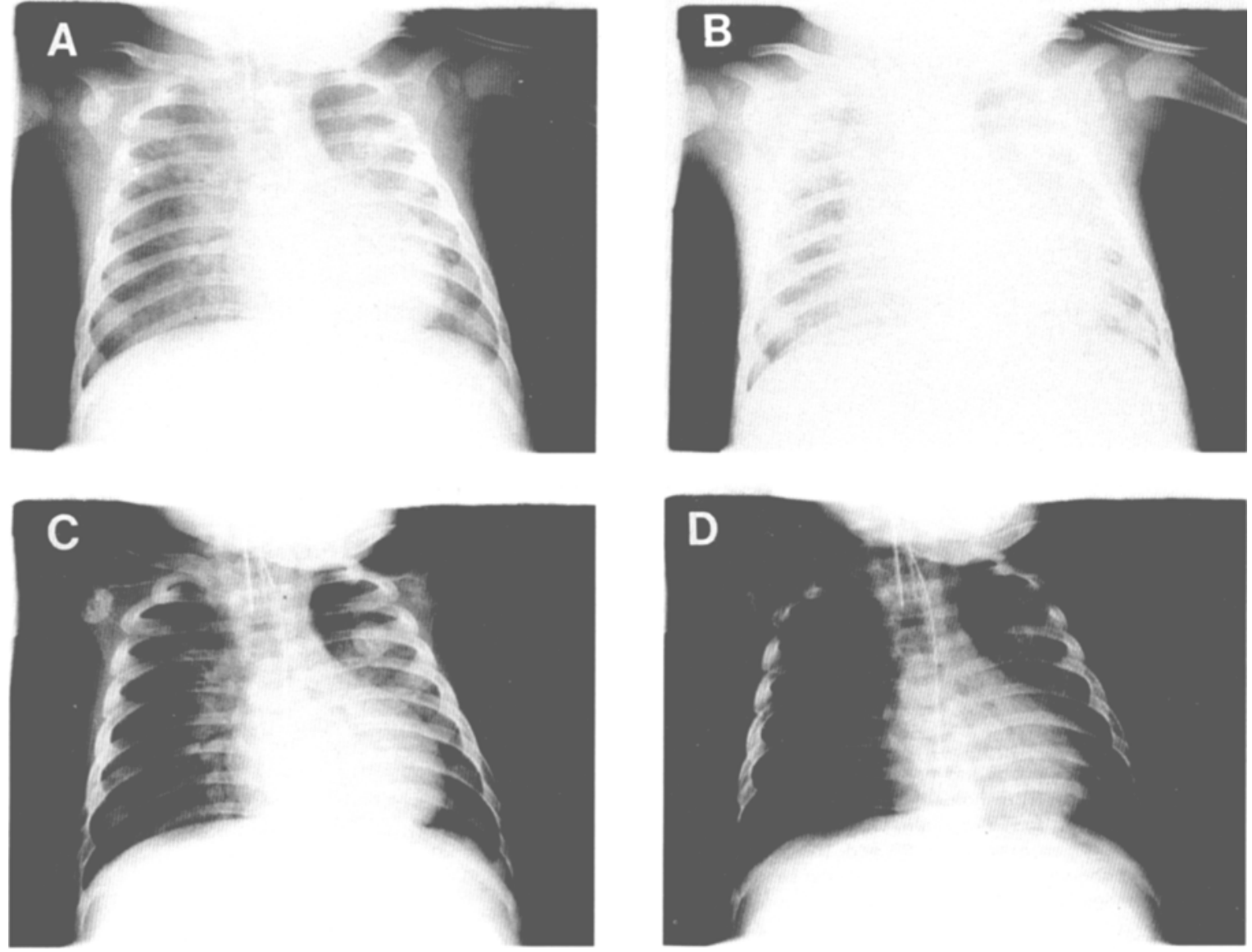

Fig 10. (A) Original digital chest radiograph of fig 1 (reproduced for comvenience). (B) LUT with soft gain of 1.4 applied to imege in Fig 10A. (C) LUT with demae gain of 1.6 applied to image in Fig 10A. (D) LUT with dense gain of 2.2 applied to image in Fig $10 A$.

diac area, the lungs (when under penetrated), and any tubes present are better visualized when the dense tissue region is enhanced. With dense tissue enhancement, lesions in the hilar, subdiaphragmatic, or retrocardiac arca may become more visible. The soft tissue enhancement does not show such dramatic improvement, but this should enhance the perception of abnormalities that may occur in the muscle, fat. or skin. This enhancement may also provide better visualization in those parts of the lung region that are over penctrated in the original exposure.

This method has been implemented in a clinical picture and archiving and communication system (PACS) since September 1991 at UCLA. ${ }^{18}$ Though no quantitative studies have been undertaken. the response to the LUTs and the flexibility provided by the multiple LUTs has been favorable.

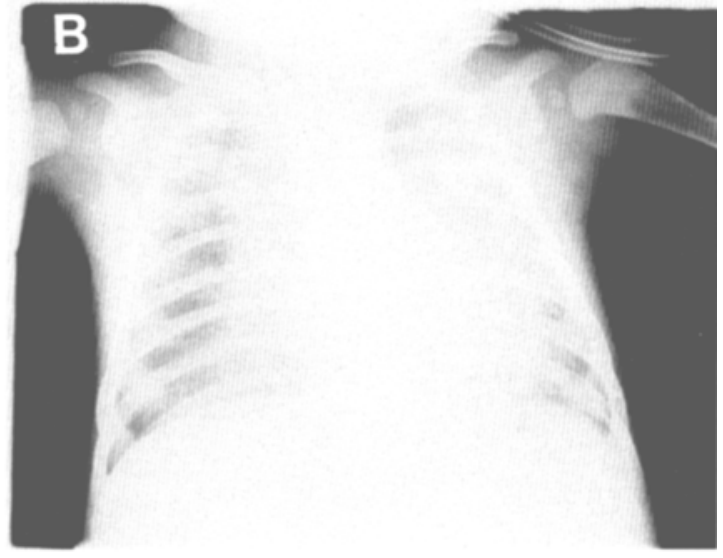

Increasing the contrast between tissues by using the LUTs may lead to an enhancement of the image noise as well. As the contrast between gray levels due to different tissue densities increases, the contrast between gray levels that are different due to noise is enhanced as welt. For the phantom image (obtained with very low $x$-ray exposure), these noise effects can be made visible. Howcver, this problem does not appear to be significant for the clinical radiographs at the levels of enhancement discussed in this report. Still. further investigation on the effects of noise are warranted. Presently, the upper limit on the individual gains is determined by the number of gray levels available for allocation. not by the amount of noise present.

Finally, this method does assume a certain shape of the histogram and that the breakpoints found do approximate the true gray level breakpoints between the anatomic regions. Thus, 


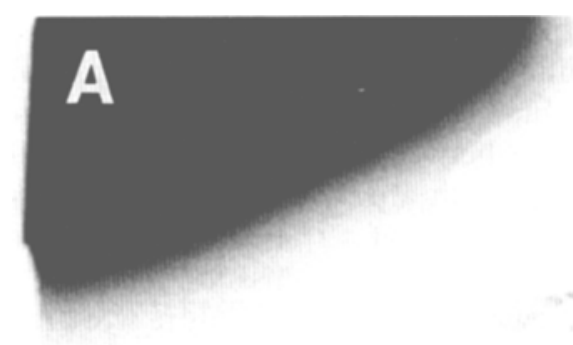

Fig 11. (A) Original image (lin. ear LUT) of chest phantom under noisy exposure conditions. (B) LUT with dense gain of 2.2 applied to image in Fig 11A to enhance the noise.
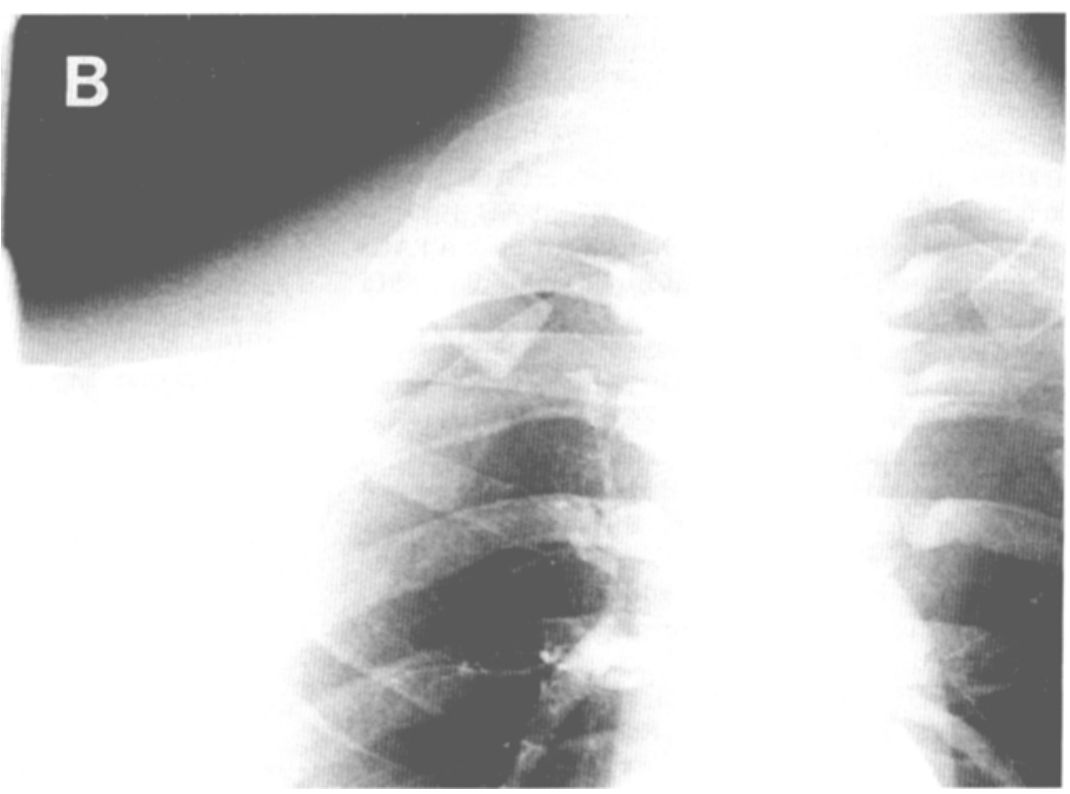

when the lung region is very dense and/or underpenetrated and the region appears bright, pixels from the lung region may be included in the radiographically dense region instead of the soft tissue region. This effect can be seen in Figs $9 \mathrm{~B}$ and $9 \mathrm{C}$ where the denser parts of the left lung region are included in the radiographically dense region. However, even if the breakpoints are not exactly correct, the LUTs created still give a great deal of flexibility in viewing the image. Even if the densest part of the lung is considered to be radiographically dense tissue, the contrast of that brighter lung region will be enhanced with the dense LUTs instead of with the soft LUTs. Therefore, even when the breakpoints are not as accurate as we would like, the overall objective-enhancement of the various tissues-can still be accomplished.

\section{CONCLUSION}

The method described in this report has the ability to enhance both soft tissues and dense tissues at the option of the viewer. Each region 
is enhanced separately. The purpose of this method is to allow the viewer to enhance either the dense tissues or the soft tissues, but not both simultaneously. The viewer can step between dense and soft tissue enhancement if both are desired. Also, the original may be accessed at any time for a direct comparison.

The option of having several piecewise linear LUTs for CR chest images has received a favorable response. Our clinicians have observed CR images that are too bright and/or lack contrast. An approach to make a single correction ${ }^{19}$ was tried, but that approach was not flexible enough for a wide variety of patients and radiologists. This new technique includes a series of LUTs to provide some flexibility. The user interface makes it extremely easy and fast to change LUTs until the desired one is found. The only drawback observed so far is that when radiographically soft tissues are enhanced, it is at the expense of decreased contrast and increased brightness of the denser tissues; and when radiographically dense tissues are enhanced, it is at the expense of decreased contrast and decreased brightness of the softer tissues.

Future work in this area may be to assess the ability of the method to standardize the display of different images of the same region having different exposure techniques. Though CR images use some processing at the time of acquisition to compensate for exposure, this is sometimes not adequate and the result is the bright and/or low contrast images. This method could possibly be used to postprocess the image to a standard display. Other work may also quantify the radiologist's response to the effectiveness (and convenience) of the LUTs in aiding diagnosis.

\section{REFERENCES}

1. Ho BK, Morioka C, Mankovich NJ, et al: Image acquisition for the pediatric PACS module. Proc SPIE 767:554-557, 1987

2. Stewart BK, Taira RK, Cho PS, et al: PACS module communication at UCLA. Proc SPIE 767:558-563, 1987

3. Kangarloo H, Boechat MI, Dietrich RB, et al: A PACS module for pediatric radiology-Current status. Proc SPIE 767:548-553, 1987

4. Taira RK, Mankovich NJ. Boechat MI, et al: Design and implementation of a picture archiving and communication system for pediatric radiology. Am J Roentgenol 150:1117-1121, 1988

5. Kangarloo H, Boechat MI, Barbaric Z, et al: Two-year clinical experience with a computed radiography system. Am J Roentgenol 151:605-608, 1988

6. Lou SL, Huang HK, Mankovich NJ, et al: A CT/ MR/US picture archiving and communication system. Proc SPIE 1093:31-36, 1989

7. Huang HK, Lou SL, Cho PS, et al: Radiologic image communication methods. Am J Roentgenol 155:183-186, 1990

8. Weinberg WS, Hayreptian A, Cho PS, et al: X Windowbased $2 k$ display workstation. Proc SPIE 1446:35-39, 1990

9. Johnson GA, Danieley N, Ravin CE: Processing alternatives for digital chest images. Radiol Clin North Am 23:244-249, 1985

10. Gonzalez RC, Wintz P: Digital Image Processing. Reading, MA, Addison-Wesley, 1987
11. Pizer SM, Zimmerman JB, Staab EV: Adaptive grey level assignment in CT scan display. J Comput Assist Tomogr 8:300-305, 1984

12. Pizer SM, Amburn EP, Austin JD, et al: Adaptive histogram equalization and its variations. Comput Vision Graph Image Processing 39:355-368, 1978

13. Rehm K, Seeley GW, Dallas WJ, et al: Design and testing of adaptive histogram equalization: A contrastenhancement technique for the display of digital chest radiographs. J Thorac Imag 5:85-91, 1990

14. McAdams HP, Johnson GA, Suddarth SA, et al: Histogram directed processing of digital chest images. Invest Radiol 21:253-259, 1986

15. Sherrier RH, Johnson GA: Regionally adaptive histogram equalization of the chest. IEEE Trans Med Imaging 6:1-7, 1987

16. Sezan MI, Tekalp AM, Schaetzing R: Automatic anatomically selective image enhancement in digital chest radiography. IEEE Trans Med Imaging 8:154-162, 1989

17. Pietka E, McNitt-Gray MF, Aberle D, et al: Computerized approach to the detection of lung nodules. Radiology 181:308, 1991 (abstr)

18. McNitt-Gray MF, Pietka E, Huang HK: Image preprocessing for a picture archiving and communication system. Invest Radiol 27:529-535, 1992

19. McNitt-Gray MF, Taira RK, Eldredge S, et al: Automatic brightness and contrast adjustments for digital chest radiographs. Radiology 177:188, 1990 (abstr) 